\section{Processing Tomato Yield and Fruit Quality Improved with Potassium Fertigation}

\author{
T.K. Hartz, P.R. Johnstone, D.M. Francis, ${ }^{1}$ and E.M. Miyao ${ }^{2}$ \\ Department of Plant Sciences, University of California, Davis, CA 95616
}

Additional key words. Lycopersicon esculentum Mill., soil fertility, soil exchangeable K, soluble solids, fruit color, foliar fertilization

\begin{abstract}
The effect of $\mathbf{K}$ fertigation through subsurface irrigation lines on processing tomato (Lycopersicon esculentum Mill.) fruit yield and quality was evaluated in four field trials in California from 2002-04. Fields had exchangeable soil $\mathrm{K}$ between 0.48 to 0.85 $\mathrm{cmol} \cdot \mathrm{kg}^{-1}$, with high exchangeable $\mathrm{Mg}\left(10.6\right.$ to $\left.13.7 \mathrm{cmol} \cdot \mathrm{kg}^{-1}\right)$ and a history of yellow shoulder (YS, a fruit color disorder) occurrence. $K$ treatments evaluated included seasonal amount applied ( 0 to $800 \mathrm{~kg} \cdot \mathrm{ha}^{-1}$ ), fertigation method (continuous versus weekly), and timing (early, mid or late season); foliar K treatments were also included in the 2002 trial. In two fields total and marketable fruit yield were significantly increased by $\mathrm{K}$ fertigation, and fruit color improvements were observed in all trials. Among color parameters improved by $\mathrm{K}$ fertigation were YS incidence, blended color, and $L^{*}$, chroma, and hue of the shoulder region of fruit. $K$ fertigation did not affect fruit soluble solids concentration. Yield increased only with fertigation treatments initiated during early fruit set. The effects of fertigation method and rate were inconsistent. Foliar $K$ application was ineffective in increasing either fruit yield or quality.
\end{abstract}

Successful processing tomato production requires both high yield and good fruit quality. The primary quality criteria for tomato paste production are blended color and soluble solids concentration (SSC), while for peeled and diced products both blended color and color uniformity are important. Potassium nutrition has been linked to tomato yield (Hartz et al., 2001; Lachover, 1972), SSC (Lachover, 1972) and fruit color (Hartz et al., 1999; Picha and Hall, 1981; Trudel and Ozbun, 1971). Uneven ripening of tomatoes is a common problem, in California and elsewhere (Francis et al., 2000; Hartz et al., 1999; Picha and Hall, 1981). A disorder called yellow shoulder(YS), in which a ring of tissue around the stem scar remains yellow after ripening, can occur at a sufficiently high frequency to render whole fields unsuitable for peel and dice processing.

In a survey of 140 commercial fields throughout central California, Hartz et al. (1999) found YS incidence to be negatively correlated with exchangeable soil $\mathrm{K}$, and positively correlated with exchangeable soil $\mathrm{Mg}$. Field trials using traditional fertilizer application techniques (preplant incorporation or sidedress banding) showed that $\mathrm{K}$ fertilization suppressed, but did not eliminate YS (Hartz et al., 1999, 2001). Fertilization rates in excess of $500 \mathrm{~kg} \cdot \mathrm{ha}^{-1}$ proved insufficient to reduce YS incidence to acceptable levels in some problem fields. One reason for such disappointing results was that many California soils have high $\mathrm{K}$ fixation characteristics; Hartz et al. (2002) and

Received for publication 12 Apr. 2005. Accepted for publication 14 may 2005 . We gratefully acknowledge the support of the USDA Initiative for Future Agriculture and Food Systems (IFAFS) program and the California Department of Food and Agriculture Fertilizer Research and Education Program (FREP).

${ }^{1}$ Ohio State University.

${ }^{2}$ University of California Cooperative Extension.
Cassman et al. (1990) showed that as much as $80 \%$ of applied $\mathrm{K}$ can be fixed in interlayer sites in vermiculitic minerals. Another is that the determinate processing cultivars used for mechanical harvesting develop smaller root systems than semi-determinate cultivars (Widders and Lorenz, 1979). This suggests that, since effective $\mathrm{K}$ diffusion is limited in most soils, the placement of $\mathrm{K}$ fertilizer in relation to the active root zone may affect crop response. The very high rates of $\mathrm{K}$ fertilization required to maximize fruit quality using traditional application techniques would be economically impractical unless significant yield increase was also achieved. However, fruit yield response to preplant or sidedress $\mathrm{K}$ application was observed only in fields with exchangeable soil $\mathrm{K}<0.35 \mathrm{cmol} \cdot \mathrm{kg}^{-1}$, while fruit color disorders commonly occurred in fields with much higher soil K supply (Hartz et al., 2001).

An alternative approach to $\mathrm{K}$ application would be $\mathrm{K}$ fertigation via drip irrigation. Since soil K fixation occurs predominately during the drying cycle following irrigation or rainfall (as K becomes more concentrated in the remaining soil solution, Cassman et al., 1990), drip irrigation may minimize fixation by maintaining more uniform soil moisture. Also, by repeatedly increasing soil solution $\mathrm{K}$ in the concentrated root zone around the drip line, frequent fertigation may significantly enhance $\mathrm{K}$ availability. This project was undertaken to investigate the effects of continuous and periodic $\mathrm{K}$ fertigation on fruit yield and quality of drip-irrigated processing tomatoes.

\section{Materials and Methods}

Four field trials were conducted in central California from 2002-04. At each site the soil exchangeable cation composition (surface 30 $\mathrm{cm}$ ) was determined by ammonium acetate extraction and analysis by atomic emission spectrometry (Thomas, 1982). All fields had a history of YS incidence, despite moderate to high exchangeable soil $\mathrm{K}(0.48$ to 0.85 $\mathrm{cmol} \cdot \mathrm{kg}^{-1}$, Table 1$)$, presumably related to the high levels of exchangeable Mg (Hartz et al., 1999). In all fields tomatoes were grown on $1.5 \mathrm{~m}$ wide raised soil beds with one drip irrigation tape buried 20 to $30 \mathrm{~cm}$ deep in the center of each bed.

The initial trial was conducted at the University of California-Davis (UCD) in 2002. The field was a Yolo silt loam (fine-silty, mixed, nonacid, thermic Typic Xerorthents). The experimental design was a split plot within a randomized complete block, with the main plots representing $\mathrm{K}$ treatment, the split plots representing cultivar. The three cultivars used ('Halley', 'Heinz 9423', and 'Peto 696') were chosen to represent a range of YS susceptibility, with 'Halley' the least, and 'Peto 969' the most susceptible. Individual experimental units were one bed wide and $25 \mathrm{~m}$ long, with five replications. The transplants were established with sprinkler irrigation on 24 Apr., with drip irrigation initiated 3 June. Drip irrigation was applied three times per week until 9 Aug, with irrigation volume determined by reference evapotranspiration (ET, modified Penman) and crop canopy development. No precipitation was received during the growing season. $\mathrm{N}$ and $\mathrm{P}$ fertility was managed by a preplant application of $33 \mathrm{~kg} \cdot \mathrm{ha}^{-1} \mathrm{P}$ and $11 \mathrm{~kg} \cdot \mathrm{ha}^{-1} \mathrm{~N}$ followed by six weekly applications of 28 $\mathrm{kg} \cdot \mathrm{ha}^{-1} \mathrm{~N}$ begun at flowering. There was no preplant $\mathrm{K}$ application.

The $\mathrm{K}$ treatments were initiated either 3 June or 24 June, corresponding to early and late fruit setting stage, respectively. The factors evaluated included foliar application vs. fertigation, continuous vs. weekly fertigation, and fertigation timing; details of the treatments evaluated are given in Table 2. Fertigated K (from $\mathrm{K}_{2} \mathrm{SO}_{4}$ ) was injected into the irrigation water by water-powered proportional injectors. All treatments received the same irrigation volume. Foliar applications of $\mathrm{K}_{2} \mathrm{SO}_{4}$ were applied with a $\mathrm{CO}_{2}$-powered sprayer. Foliar sprays contained $0.125 \%$ nonionic surfactant and were applied at $150 \mathrm{~L} \cdot \mathrm{ha}^{-1}$ on a broadcast basis; $\mathrm{K}$ concentration in the spray was $48 \mathrm{~g} \cdot \mathrm{L}^{-1}$.

To document the $\mathrm{K}$ status of the crop, samples of most-recently matured leaves were collected in each experimental unit on 25 June and 21 July, corresponding to late fruit set and early red fruit developmental stages, respectively. The leaves were rinsed to remove surface spray residue, oven-dried, ground, and analyzed for $\mathrm{K}$ concentration by atomic emission spectrometry following extraction in $2 \%$ acetic acid (Johnson and Ulrich, 1959). To mimic the normal commercial practice, a single destructive fruit harvest was conducted when each cultivar reached approximately $95 \%$ red fruit. Harvest dates were 20, 22, and 28 Aug. for 'Heinz 9423', 'Peto 696', and 'Halley', respectively. Total fruit yield, and yield of sound, red fruit (marketable yield) was determined. A fruit sample from each experimental unit was juiced and evaluated for soluble solids 
concentration (SSC, ${ }^{\circ}$ brix, by refractometer) and blended color [ratio of green $(566 \mathrm{~nm})$ to red $(650 \mathrm{~nm})$ light reflected from the juice]. In total, 30 red fruit from each experimental unit were visually evaluated for YS. Additionally, the peel was removed from the shoulder region of 20 red fruit per experimental unit, and these fruit were evaluated for color of the exposed pericarp using a colorimeter (model 300; Minolta Co., Ramsey, N.J.). Two measurements of $\mathrm{L}^{*}$, chroma, and hue were made on each fruit. $\mathrm{L}^{*}$ is the lightness coefficient, chroma a color saturation index, and hue a measure of redness (McGuire, 1992).

A second field trial was conducted at UCD in 2003. The soil was a Yolo silt loam, with physiochemical characteristics similar to the 2002 trial site (Table 1). The experimental design and cultivars used were the same as in the 2002 experiment, although the $\mathrm{K}$ treatments were different (Table 2). Transplants were established with sprinkler irrigation on 13 May. Drip irrigation began 18 June and water was applied three times per week until irrigation cutoff on $20 \mathrm{Aug}$. Irrigation volume was determined by reference evapotranspiration(ET, modified Penman) and crop canopy development. $\mathrm{N}$ and $\mathrm{P}$ fertility was managed as described for 2002 .

The $\mathrm{K}$ fertigation treatments were initiated on 18 June, 2 July, or 16 July, spanning the main fruit setting period. The factors evaluated included continuous vs. weekly $\mathrm{K}$ fertigation, and fertigation timing. Fertigated K was injected into the irrigation water by waterpowered proportional injectors; all treatments received the same irrigation volume. Potassium chloride $(\mathrm{KCl})$ was used as the $\mathrm{K}$ source for all fertigation treatments; the field received no preplant $\mathrm{K}$ application. Whole-leaf samples for K analysis were collected on 11 July and 1 Aug., corresponding to late fruit set and early red fruit developmental stages, respectively. A single destructive fruit harvest was conducted when each cultivar reached approximately $95 \%$ red fruit. Harvest dates were 28 Aug., 2 Sept., and 4 Sept. for 'Heinz 9423', 'Peto 696', and 'Halley', respectively. Fruit yield and quality were evaluated as described for the 2002 trial, with the exception that 25 fruit per experimental unit, rather than 20 , were examined for pericarp color. Additionally, red fruit from each experimental unit were oven-dried, ground, and analyzed for $\mathrm{K}$ concentration.

In 2004, two trials were conducted in dripirrigated commercial fields near Winters and Woodland. The soil at both sites was a Capay silty clay (fine, montmorillonitic, thermic Typic Chromoxererts, Table 1). The Winters field was transplanted with 'Heinz 2601' plants 18 Mar., and the Woodland field was transplanted with 'Heinz 9780' plants 16 Apr. At each site two $\mathrm{K}$ fertigation treatments were evaluated: 1) continuous $\mathrm{K}$ fertigation at $100 \mathrm{mg} \cdot \mathrm{L}^{-1} \mathrm{~K}$, and 2) five weekly fertigations of $45 \mathrm{~kg} \cdot \mathrm{ha}^{-1} \mathrm{~K}$. Potassium chloride was the $\mathrm{K}$ source for both treatments at both sites. The continuous fertigation treatment began on 13 May at Winters and 26 May at Woodland; crop growth stage was early- and mid-fruit set at Woodland and Winters, respectively. Continuous fertigation was discontinued after a seasonal total of 225 $\mathrm{kg} \mathrm{K} /$ ha had been applied; this occurred on 30 June at Winters and 28 June at Woodland, at early red fruit stage. In the weekly fertigation treatment $\mathrm{K}$ was applied in a single irrigation per week for 5 consecutive weeks, for a seasonal application of $225 \mathrm{~kg} \mathrm{~K} / \mathrm{ha}$. Weekly fertigation began on 21 May at Winters and 3 June at Woodland. For each fertigation treatment a pump operated by a pressure-sensitive switch (which turned on when the irrigation system was pressurized) pumped the $\mathrm{KCl}$ into the irrigation stream of individual drip lines; all treatments received the same amount of water and $\mathrm{N}$ fertilizer applied by the grower; the only difference was the addition of $\mathrm{KCl}$ in

selected treatments. A randomized complete block experimental design with six replications was used at both sites, comparing the $\mathrm{K}$ fertigation treatments and the grower irrigation treatment receiving no $\mathrm{K}$ fertigation. Individual plots were one bed wide by either $100 \mathrm{~m}$ (Woodland) or $400 \mathrm{~m}$ long (Winters). The growers applied a seasonal total of 215 and $280 \mathrm{~kg} \cdot \mathrm{ha}^{-1} \mathrm{~N}$, and 47 and $35 \mathrm{~kg} \cdot \mathrm{ha}^{-1} \mathrm{P}$ at Winters and Woodland, respectively. At Winters $120 \mathrm{~kg} \cdot \mathrm{ha}^{-1} \mathrm{~K}$ had been applied preplant; soil sampling was done after transplanting, so the effect of that application was reflected in the exchangeable $\mathrm{K}$ value listed in Table 1. At Woodland the grower made no preplant or in-season $\mathrm{K}$ application.

Whole-leaf samples were collected on 11 and 29 June at Winters and 23 June and 7 July at Woodland, corresponding to late fruit set and early red fruit developmental stage, respectively. Leaf samples were also analyzed for total $\mathrm{N}$ by a combustion technique (Dumas, 1981) and P by ICP-AES following microwave acid digestion (Sah and Miller, 1992). At commercial maturity a 30-m section in the middle of each plot was machine-harvested. Fruit yield and quality were evaluated as described for the 2003 trial.

\section{Results}

In 2002, continuous K fertigation increased late-season foliar $\mathrm{K}$ concentration, while neither weekly fertigation nor foliar application had a significant effect (Table 3 ). The superiority of continuous injection undoubtedly reflected the very high seasonal application rates employed. The $100 \mathrm{mg} \cdot \mathrm{L}^{-1}$ continuous $\mathrm{K}$

Table 1. Soil characteristics at the $\mathrm{K}$ fertigation trial sites.

\begin{tabular}{llllrl}
\hline & & & & \multicolumn{2}{c}{$\begin{array}{c}\text { Soil exchangeable } \\
\text { cations }\left(\mathrm{cmol}^{-1} \mathrm{~kg}^{-1}\right)\end{array}$} \\
\cline { 4 - 6 } Year & Location & Soil & & $\mathrm{Ca}$ & $\mathrm{Mg}$ \\
\hline 2002 & UCD $^{z}$ & silt loam & 0.85 & 7.8 & 12.0 \\
2003 & UCD & silt loam & 0.84 & 8.0 & 10.8 \\
2004 & Winters & silty clay & 0.48 & 15.7 & 10.6 \\
& Woodland & silty clay & 0.70 & 14.0 & 13.7 \\
\hline
\end{tabular}

${ }^{\mathrm{z}} \mathrm{UCD}=$ University of California-Davis.

Table 2. Description of K fertigation and foliar spray treatments, 2002 and 2003 University of California-Davis trials.

\begin{tabular}{|c|c|c|c|}
\hline $\mathrm{K}$ treatment & $\begin{array}{c}\text { Date } \\
\text { begun }\end{array}$ & Description & $\begin{array}{c}\text { Seasonal K } \\
\text { application } \\
\left(\mathrm{kg} \cdot \mathrm{ha}^{-1}\right)\end{array}$ \\
\hline \multicolumn{4}{|l|}{2002} \\
\hline Fertigation continuously $\left(100 \mathrm{mg} \cdot \mathrm{L}^{-1}\right)$ & 3 June & $\mathrm{K}$ continuously injected until 9 Aug & 400 \\
\hline Fertigation continuously $\left(200 \mathrm{mg} \cdot \mathrm{L}^{-1}\right)$ & 3 June & K continuously injected until 9 Aug & 800 \\
\hline Early weekly fertigation & 3 June & 5 weekly $\mathrm{K}$ injections at $50 \mathrm{~kg} \cdot \mathrm{ha}^{-1} \mathrm{~K}$ & 250 \\
\hline Late weekly fertigation & 24 June & 5 weekly $\mathrm{K}$ injections at $50 \mathrm{~kg} \cdot \mathrm{ha}^{-1} \mathrm{~K}$ & 250 \\
\hline Early weekly foliar spray & 3 June & 5 weekly applications of $7.2 \mathrm{~kg} \cdot \mathrm{ha}^{-1} \mathrm{~K}$ & $29^{z}$ \\
\hline Late weekly foliar spray & 24 June & 5 weekly applications of $7.2 \mathrm{~kg} \cdot \mathrm{ha}^{-1} \mathrm{~K}$ & 36 \\
\hline No K controly & & & 0 \\
\hline \multicolumn{4}{|l|}{2003} \\
\hline Continuously, full season $\left(100 \mathrm{mg} \cdot \mathrm{L}^{-1}\right)$ & 18 June & K continuously injected until 20 Aug & 440 \\
\hline Continuously, full season $\left(50 \mathrm{mg} \cdot \mathrm{L}^{-1}\right)$ & 18 June & K continuously injected until 20 Aug & 220 \\
\hline Continuously, midseason only $\left(100 \mathrm{mg} \cdot \mathrm{L}^{-1}\right)$ & 2 July & K continuously injected until 8 Aug & 320 \\
\hline Continuously, midseason only $\left(200 \mathrm{mg} \cdot \mathrm{L}^{-1}\right)$ & 2 July & K continuously injected until 8 Aug & 640 \\
\hline Early weekly fertigation & 18 June & 5 weekly $\mathrm{K}$ applications of $45 \mathrm{~kg} \cdot \mathrm{ha}^{-1}$ & 225 \\
\hline Midseason weekly fertigation & 2 July & 5 weekly applications of $45 \mathrm{~kg} \cdot \mathrm{ha}^{-1}$ & 225 \\
\hline Late weekly $\mathrm{K}$ fertigation & 16 July & 5 weekly applications of $45 \mathrm{~kg} \cdot \mathrm{ha}^{-1}$ & 225 \\
\hline No K control & & & 0 \\
\hline
\end{tabular}

${ }^{2}$ Early foliar treatment had smaller plants on the early application dates, limiting sprayed area.

${ }^{y}$ No foliar or fertigated $\mathrm{K}$. 
treatment had been intended to deliver approximately the same total $\mathrm{K}$ amount as the batch application treatments, but abnormally high seasonal $\mathrm{ET}_{\mathrm{o}}$ and unexpectedly vigorous vine growth increased the irrigation requirement,

and hence the seasonal $\mathrm{K}$ application.

Potassium fertigation significantly improved fruit yield and quality, while foliar $\mathrm{K}$ was ineffective (Table 4 and 5). Continuous fertigation increased total fruit yield of 'Halley'

Table 3. Effect of $\mathrm{K}$ treatment on leaf $\mathrm{K}$ concentration at late fruit set (25 June) and early red fruit stage (21 July), 2002 University of California-Davis trial.

\begin{tabular}{|c|c|c|c|}
\hline \multirow[b]{2}{*}{$\mathrm{K}$ treatment } & \multirow{2}{*}{$\begin{array}{c}\text { Seasonal K } \\
\left(\mathrm{kg} \cdot \mathrm{ha}^{-1}\right)\end{array}$} & \multicolumn{2}{|c|}{ Leaf K $\left(\mathrm{g} \cdot \mathrm{kg}^{-1}\right)$} \\
\hline & & 25 June & 21 July \\
\hline Continuously $\left(100 \mathrm{mg} \cdot \mathrm{L}^{-1}\right)$ & 400 & 30.9 & $18.7 \mathrm{a}^{2}$ \\
\hline Continuously $\left(200 \mathrm{mg} \cdot \mathrm{L}^{-1}\right)$ & 800 & 32.1 & $17.6 \mathrm{a}$ \\
\hline Early weekly fertigation & 250 & 31.9 & $12.7 \mathrm{~b}$ \\
\hline Late weekly fertigation & 250 & 29.5 & $13.3 \mathrm{~b}$ \\
\hline Early weekly foliar spray & 29 & 31.8 & $11.9 \mathrm{~b}$ \\
\hline Late weekly foliar spray & 36 & 29.6 & $13.3 \mathrm{~b}$ \\
\hline No K control & 0 & 29.5 & $9.7 \mathrm{~b}$ \\
\hline Treatment $\times$ cultivar interaction & & NS & NS \\
\hline \multicolumn{4}{|l|}{ Contrasts } \\
\hline Fertigation vs. no K control & & NS & $* *$ \\
\hline Foliar spray vs. no K control & & $*$ & NS \\
\hline Foliar vs. fertigation & & $*$ & $* *$ \\
\hline Continuous vs. weekly fertigation & & NS & $* *$ \\
\hline \multicolumn{4}{|l|}{ Cultivar } \\
\hline 'Halley’ & & $29.7 \mathrm{~b}$ & $15.7 \mathrm{a}$ \\
\hline 'Heinz 9423' & & $28.9 \mathrm{~b}$ & $13.0 \mathrm{~b}$ \\
\hline 'Peto 696' & & $33.6 \mathrm{a}$ & $13.0 \mathrm{~b}$ \\
\hline
\end{tabular}

${ }^{\mathrm{z}}$ Means within columns separated using Duncan's multiple range test, $p<0.05$.

NS, ${ }^{*, * *}$ Nonsignificant or significant at $p<0.05$ or 0.01 , respectively. and 'Peto 696', with early weekly fertigation also improving total yield of 'Peto 696'; marketable yield of 'Halley' and 'Peto 696' was increased by all three of those treatments. Yield increase was primarily attributable to increased fruit number, as there were no significant differences in mean fruit mass (data not shown). Effects on SSC were variable, with the fertigated treatments having slightly lower brix. This appeared to be an example of the commonly observed 'dilution' effect of high yield (Dumas et al., 1994) rather than any reduction of solids yield, since the brix yield (marketable yield $\times{ }^{\circ}$ brix) was highest in the highest yielding treatments. Blended fruit color varied over a narrow range, with only the contrast between continuous and weekly fertigation significant. Blended color was a dimensionless unit, with lower values representing redder fruit.

YS incidence varied by cultivar, with 'Peto 696' exhibiting more than twice the percentage of affected fruit compared to the other cultivars. All fertigation treatments were equally effective in reducing YS. Pericarp $\mathrm{L}^{*}$ value and hue were most positively influenced by continuous K injection, with late-season batch application also having a significant positive

Table 4. Effect of K treatment on tomato fruit yield, soluble solids concentration (SSC), and color quality, 2002 University of California-Davis trial.

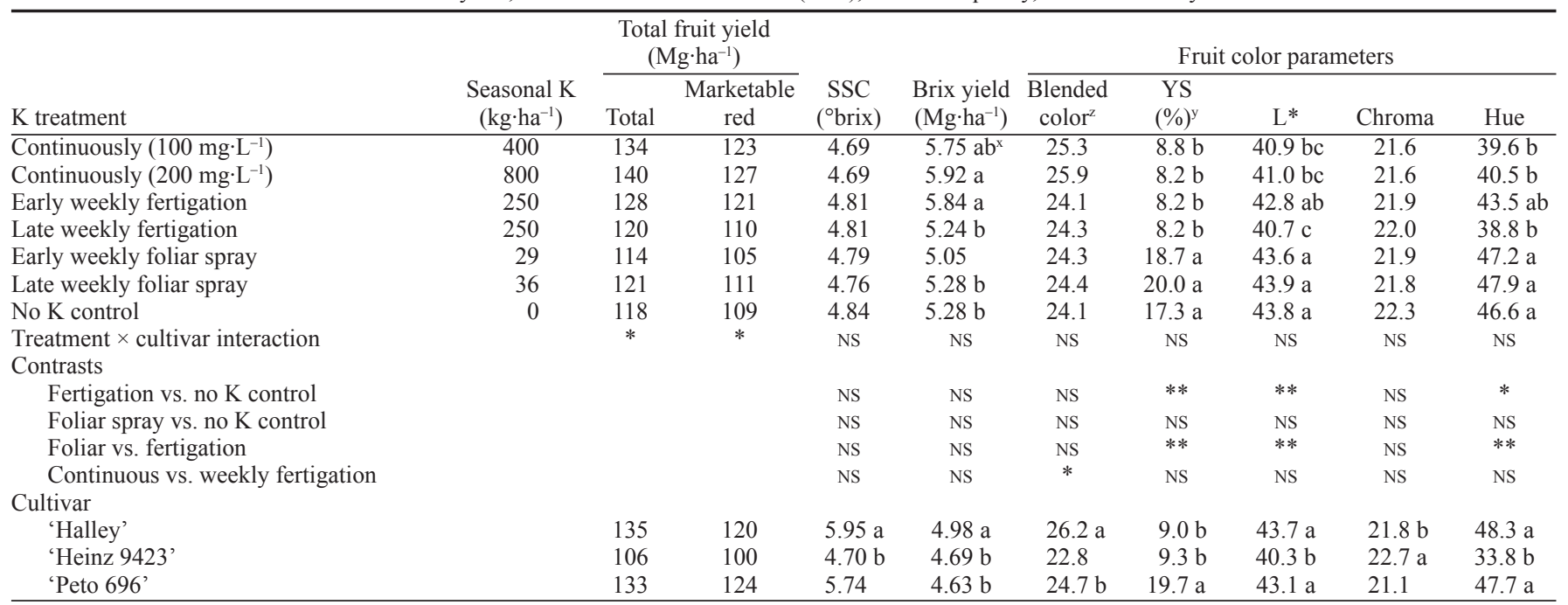

${ }^{2}$ Dimensionless unit, lower value indicates more red

yS = yellow shoulder.

${ }^{x}$ Means within columns separated using Duncan's multiple range test, $p<0.05$.

NS,*,**N Nonsignificant or significant at $p<0.05$ or 0.01 , respectively.

Table 5. Cultivar-specific effects of K treatment on tomato fruit yield, 2002 UCD trial.

\begin{tabular}{|c|c|c|c|c|c|c|c|}
\hline \multirow[b]{2}{*}{$\mathrm{K}$ treatment } & \multirow{2}{*}{$\begin{array}{c}\text { Seasonal K } \\
\left(\mathrm{kg} \cdot \mathrm{ha}^{-1}\right)\end{array}$} & \multicolumn{3}{|c|}{ Total fruit yield $\left(\mathrm{Mg} \cdot \mathrm{ha}^{-1}\right)$} & \multicolumn{3}{|c|}{ Marketable fruit yield $\left(\mathrm{Mg} \cdot \mathrm{ha}^{-1}\right)$} \\
\hline & & 'Halley' & 'Heinz 9423' & 'Peto 696' & 'Halley' & 'Heinz 9423' & 'Peto 696 \\
\hline Continuously $\left(200 \mathrm{mg} \cdot \mathrm{L}^{-1}\right)$ & 800 & $157 \mathrm{a}$ & 107 & $150 \mathrm{a}$ & $136 \mathrm{a}$ & 101 & $138 \mathrm{a}$ \\
\hline Early weekly foliar spray & 29 & 118 & 102 & 123 & 104 & 97 & $115 \mathrm{c}$ \\
\hline Late weekly foliar spray & 36 & 129 & 102 & $130 \mathrm{~b}$ & $112 \mathrm{~b}$ & 98 & $120 \mathrm{bc}$ \\
\hline No K control & 0 & 124 & 107 & 123 & 108 & 102 & $117 \mathrm{c}$ \\
\hline Foliar spray vs. no K control & & NS & NS & NS & NS & NS & NS \\
\hline Foliar vs. fertigation & & ** & NS & ** & ** & NS & ** \\
\hline Continuous vs. weekly fertigation & & $* *$ & NS & $* *$ & * & NS & $* *$ \\
\hline
\end{tabular}

${ }^{\mathrm{z}}$ Means within columns separated using Duncan's multiple range test, $p<0.05$.

${ }_{\mathrm{NS}, *, * *}$ Nonsignificant or significant at $p<0.05$ or 0.01 , respectively. 
Table 6. Effect of $\mathrm{K}$ treatment on leaf $\mathrm{K}$ concentration $\left(\mathrm{g} \cdot \mathrm{kg}^{-1}\right)$ at late fruit set (11 June) and early red fruit stage (1 Aug.), $2003 \mathrm{University} \mathrm{of} \mathrm{California-Davis} \mathrm{trial.}$

\begin{tabular}{|c|c|c|c|c|c|c|}
\hline \multirow[b]{2}{*}{$\mathrm{K}$ treatment } & \multicolumn{2}{|c|}{ 'Halley' } & \multicolumn{2}{|c|}{ 'Heinz 9423' } & \multicolumn{2}{|c|}{ 'Peto 696' } \\
\hline & 11 June & 1 Aug. & 11 June & $1 \mathrm{Aug}$. & 11 June & 1 Aug. \\
\hline Continuously, full season $\left(100 \mathrm{mg} \cdot \mathrm{L}^{-1}\right)$ & 31.7 & $15.0 \mathrm{abc}^{\mathrm{z}}$ & 31.7 & 12.8 & 36.4 & $18.4 \mathrm{ab}$ \\
\hline Continuously, full season $\left(50 \mathrm{mg} \cdot \mathrm{L}^{-1}\right)$ & 32.5 & $15.6 \mathrm{ab}$ & 30.8 & 14.1 & 35.4 & $16.9 \mathrm{ab}$ \\
\hline Continuously, midseason only $\left(100 \mathrm{mg} \cdot \mathrm{L}^{-1}\right)$ & 34.8 & $14.0 \mathrm{~b}$ & 30.5 & 14.1 & 34.6 & $15.4 \mathrm{~b}$ \\
\hline Continuously, midseason only $\left(200 \mathrm{mg} \cdot \mathrm{L}^{-1}\right)$ & 30.0 & $17.3 \mathrm{a}$ & 32.5 & 13.5 & 36.6 & $17.1 \mathrm{ab}$ \\
\hline Early weekly fertigation & 34.4 & $16.2 \mathrm{ab}$ & 31.0 & 13.6 & 35.6 & $15.2 \mathrm{~b}$ \\
\hline Midseason weekly fertigation & 32.3 & $13.8 \mathrm{~b}$ & 28.4 & 13.2 & 36.1 & $16.0 \mathrm{~b}$ \\
\hline Late weekly fertigation & 31.0 & $14.8 \mathrm{ab}$ & 30.3 & 12.9 & 36.1 & $21.1 \mathrm{a}$ \\
\hline No K control & 29.4 & 13.0 & 32.6 & 15.4 & 33.5 & $14.6 \mathrm{~b}$ \\
\hline \multicolumn{7}{|l|}{ Contrast } \\
\hline Fertigation vs. no K control & NS & * & NS & NS & NS & NS \\
\hline
\end{tabular}

${ }^{2}$ Means within columns separated using Duncan's multiple range test, $p<0.05$.

${ }^{\mathrm{NS}}$ Nonsignificant at $p<0.05$.

Table 7. Effect of K treatment on tomato fruit yield, soluble solids concentration (SSC) and color quality, 2003 University of California-Davis trial.

\begin{tabular}{|c|c|c|c|c|c|c|c|c|c|c|c|}
\hline \multirow[b]{3}{*}{$\mathrm{K}$ treatment } & \multirow{3}{*}{$\begin{array}{c}\text { Seasonal K } \\
\left(\mathrm{kg} \cdot \mathrm{ha}^{-1}\right)\end{array}$} & \multirow{2}{*}{\multicolumn{2}{|c|}{$\begin{array}{c}\text { Fruit yield } \\
\left(\mathrm{Mg} \cdot \mathrm{ha}^{-1}\right)\end{array}$}} & \multirow{3}{*}{$\begin{array}{c}\mathrm{SS} \\
\left({ }^{\circ} \text { brix }\right)\end{array}$} & \multirow{3}{*}{$\begin{array}{c}\text { Brix } \\
\text { yield } \\
\left(\mathrm{Mg} \cdot \mathrm{ha}^{-1}\right)\end{array}$} & \multicolumn{5}{|c|}{ Fruit color parameter } & \multirow{3}{*}{$\begin{array}{c}\text { Fruit K } \\
(\%)\end{array}$} \\
\hline & & & & & & \multirow{2}{*}{$\begin{array}{c}\text { Blended } \\
\text { color }^{z}\end{array}$} & \multirow{2}{*}{$\begin{array}{l}Y^{y} \\
(\%)\end{array}$} & \multirow[b]{2}{*}{$\mathrm{L}^{*}$} & \multirow[b]{2}{*}{ Chroma } & \multirow[b]{2}{*}{ Hue } & \\
\hline & & Total & Marketable & & & & & & & & \\
\hline Full season $\left(100 \mathrm{mg} \cdot \mathrm{L}^{-1}\right)$ & 440 & 101 & 80 & 4.73 & 3.78 & $24.5 \mathrm{abc}^{\mathrm{x}}$ & $10.1 \mathrm{~b}$ & $42.4 \mathrm{~b}$ & 22.6 & $41.0 \mathrm{ab}$ & 4.14 \\
\hline Full season $\left(50 \mathrm{mg} \cdot \mathrm{L}^{-1}\right)$ & 220 & 106 & 84 & 4.76 & 4.00 & $24.3 \mathrm{~b}$ & $11.7 \mathrm{~b}$ & 41.7 & 22.7 & 38.9 & 4.15 \\
\hline Midseason $\left(100 \mathrm{mg} \cdot \mathrm{L}^{-1}\right)$ & 320 & 102 & 81 & 4.85 & 3.93 & 24.0 & $9.7 \mathrm{~b}$ & 41.8 & 22.8 & 39.8 & 4.01 \\
\hline Midseason $\left(200 \mathrm{mg} \cdot \mathrm{L}^{-1}\right)$ & 640 & 102 & 80 & 4.86 & 3.89 & $24.1 \mathrm{~b}$ & $13.7 \mathrm{ab}$ & $43.1 \mathrm{ab}$ & 22.6 & $42.3 \mathrm{ab}$ & 4.06 \\
\hline Early weekly fertigation & 225 & 105 & 80 & 4.78 & 3.82 & $24.3 \mathrm{~b}$ & $14.0 \mathrm{ab}$ & $42.5 \mathrm{~b}$ & 22.8 & $40.9 \mathrm{ab}$ & 4.22 \\
\hline Midseason weekly fertigation & 225 & 107 & 84 & 4.79 & 4.02 & $25.2 \mathrm{ab}$ & $11.7 \mathrm{~b}$ & 42.1 & 22.6 & $40.4 \mathrm{~b}$ & 4.11 \\
\hline Late weekly fertigation & 225 & 103 & 80 & 4.82 & 3.86 & $25.0 \mathrm{ab}$ & $9.9 \mathrm{~b}$ & 41.7 & 22.5 & 39.2 & 4.08 \\
\hline No K control & 0 & 106 & 85 & 4.63 & 3.94 & $25.6 \mathrm{a}$ & $18.7 \mathrm{a}$ & $43.3 \mathrm{a}$ & 22.7 & $43.2 \mathrm{a}$ & 4.00 \\
\hline Treatment $\times$ cultivar interaction & & NS & NS & NS & NS & NS & NS & NS & NS & NS & NS \\
\hline \multicolumn{12}{|l|}{ Contrasts } \\
\hline Fertigation vs. no $\mathrm{K}$ control & & NS & NS & $*$ & NS & $*$ & $* *$ & $*$ & NS & $*$ & NS \\
\hline \multicolumn{12}{|l|}{ Cultivar } \\
\hline 'Halley' & & 96 & 67 & $4.91 \mathrm{a}$ & 3.29 & $25.3 \mathrm{a}$ & $11.3 \mathrm{~b}$ & $43.4 \mathrm{a}$ & $23.7 \mathrm{a}$ & $42.2 \mathrm{a}$ & $3.83 \mathrm{c}$ \\
\hline 'Heinz 9423' & & $103 \mathrm{~b}$ & $93 \mathrm{a}$ & $4.88 \mathrm{a}$ & $4.54 \mathrm{a}$ & $24.1 \mathrm{~b}$ & $8.7 \mathrm{~b}$ & 41.1 & $23.6 \mathrm{a}$ & $36.8 \mathrm{~b}$ & $4.37 \mathrm{a}$ \\
\hline 'Peto 696' & & $112 \mathrm{a}$ & $85 \mathrm{~b}$ & $4.54 \mathrm{~b}$ & $3.77 \mathrm{~b}$ & $24.5 \mathrm{~b}$ & $17.4 \mathrm{a}$ & $42.4 \mathrm{~b}$ & $20.8 \mathrm{~b}$ & $42.9 \mathrm{a}$ & $4.0 \mathrm{~b}$ \\
\hline
\end{tabular}

${ }^{2}$ Dimensionless unit, lower value indicates more red

${ }^{\mathrm{y}} \mathrm{YS}=$ yellow shoulder.

${ }^{x}$ Means within columns separated using Duncan's multiple range test, $p<0.05$.

NS,*,**N Nonsignificant or significant at $p<0.05$ or 0.01 , respectively.

Table 8. Effect of $\mathrm{K}$ treatment on leaf $\mathrm{K}$ concentration $\left(\mathrm{g} \cdot \mathrm{kg}^{-1}\right)$ at late fruit set and early red fruit stage, 2004 trials.

\begin{tabular}{lccccc}
\hline & \multicolumn{2}{c}{ Winters } & & \multicolumn{2}{c}{ Woodland } \\
\cline { 2 - 3 } \cline { 5 - 6 } \cline { 5 - 6 } K treatment & $\begin{array}{c}\text { Late fruit set } \\
\text { 11 June }\end{array}$ & $\begin{array}{c}\text { Early red fruit } \\
\text { 29 June }\end{array}$ & & $\begin{array}{c}\text { Late fruit set } \\
\text { 23 June }\end{array}$ & $\begin{array}{c}\text { Early red fruit } \\
\text { 7 July }\end{array}$ \\
\hline Continuously $\left(100 \mathrm{mg} \cdot \mathrm{L}^{-1}\right)$ & $14.0 \mathrm{~b}^{\mathrm{y}}$ & $8.0 \mathrm{a}$ & & $23.5 \mathrm{a}$ & $19.4 \mathrm{a}$ \\
Weekly fertigation & $16.3 \mathrm{a}$ & $8.4 \mathrm{a}$ & & $22.8 \mathrm{a}$ & $18.6 \mathrm{a}$ \\
No K control & $13.5 \mathrm{~b}$ & $6.7 \mathrm{~b}$ & & $17.3 \mathrm{~b}$ & $11.3 \mathrm{~b}$ \\
Contrast & & $* *$ & $* *$ & $* *$ & $* *$ \\
$\quad$ Fertigation vs. no K control & $* *$ & & $* *$ \\
\hline
\end{tabular}

${ }^{\mathrm{z}} \mathrm{A}$ seasonal total of $225 \mathrm{~kg} \cdot \mathrm{ha}^{-1} \mathrm{~K}$ was applied in both fertigation treatments.

${ }^{\mathrm{y}}$ Means within columns separated using Duncan's multiple range test, $p<0.05$.

NS, ${ }^{* * *}$ Nonsignificant or significant at $p<0.05$ or 0.01 , respectively.

effect (for L*, chroma and hue, lower values are desirable). Foliar K had no significant influence on fruit color. Chroma was not affected by any $\mathrm{K}$ treatment.

In 2003 there was a $\mathrm{K}$ treatment $\times$ cultivar interaction on leaf $\mathrm{K}$ concentration on both sampling dates. The $\mathrm{K}$ fertigation had little effect on leaf $\mathrm{K}$ on either date, with significant treatment differences limited to 'Halley' and 'Peto 696' at the late sampling date (Table 6); across treatments, leaf $\mathrm{K}$ at early red fruit stage was higher than in 2002, and well above the minimum sufficiency levels suggested by Hartz et al. (1998). There were no significant $\mathrm{K}$ treatment effects on total or marketable fruit yield (Table 7). Precipitation (about $20 \mathrm{~mm}$ ) on 22 Aug resulted in a high incidence of fruit rot, particularly in 'Halley' and 'Peto 696'; K treatment did not affect the cull rate. Evaluated collectively, the K treatments increased SSC, but given the trend toward lower yield with $\mathrm{K}$ application there were no differences in brix yield. Fruit color was improved across cultivars with K fertigation; improvement was seen in blended color, YS incidence, $\mathrm{L}^{*}$, and hue. Among the continuous fertigation treatments, $\mathrm{K}$ rate or timing had little effect. Fruit K concentration was not increased with $\mathrm{K}$ fertigation. Large differences existed among cultivars in yield and quality characteristics; as in 2002, 'Peto 696' had the greatest YS incidence.

In 2004, $\mathrm{K}$ fertigation increased leaf $\mathrm{K}$ concentration at both sites, with the two fertigation methods about equally effective (Table 8 ). Leaf $\mathrm{K}$ response was greater at Woodland, where late-season leaf $\mathrm{K}$ was nearly doubled by fertigation. At Winters, neither K treatment significantly increased fruit yield, SSC or brix yield (Table 9). The $\mathrm{K}$ fertigation did not significantly reduce YS incidence (which was low in all treatments), but did improve L value and hue; both fertigation treatments were equally effective. Leaf $P$ at Winters was substantially below established sufficiency levels at both growth stages $[0.22 \%$ and $0.16 \% \mathrm{P}$ at late fruit set and early red fruit, respectively, compared to the minimum sufficiency levels of $0.25 \%$ and $0.23 \%$ suggested by Hartz et al., (1998)], suggesting that $\mathrm{P}$ availability may have been a yield-limiting factor confounding $\mathrm{K}$ fertigation effects. At Woodland, K fertigation by either method resulted in significantly improved fruit yield, brix yield, blended color, $L^{*}$, chroma, hue and fruit K concentration. As in the 2002 UCD trial, yield increase was attributable primarily to increased fruit number, as fertigation did not significantly affect mean fruit mass ( $p>0.05$, data not shown). The apparent increase in YS with $\mathrm{K}$ fertigation was considered an anomaly in light of the significant improvement in all other color parameters with $\mathrm{K}$ fertigation, and the record of YS reduction with $\mathrm{K}$ fertigation found in the other trials.

\section{Discussion}

These results confirmed that $\mathrm{K}$ fertigation through subsurface drip irrigation was 


\begin{tabular}{|c|c|c|c|c|c|c|c|c|c|}
\hline \multirow{2}{*}{$\mathrm{K}$ treatment ${ }^{2}$} & \multirow{2}{*}{\multicolumn{2}{|c|}{$\begin{array}{c}\text { Fruit yield } \\
\left(\mathrm{Mg} \cdot \mathrm{ha}^{-1}\right)\end{array}$}} & \multirow{2}{*}{$\begin{array}{c}\text { SS } \\
\left({ }^{\circ} \text { brix }\right)\end{array}$} & \multirow{2}{*}{ 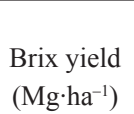 } & \multicolumn{5}{|c|}{ Fruit color parameter } \\
\hline & & & & & $\begin{array}{c}\text { Blended } \\
\text { color }^{y}\end{array}$ & $\begin{array}{l}Y^{\mathrm{x}} \\
(\%)\end{array}$ & $\mathrm{L}^{*}$ & Chroma & Hue \\
\hline \multicolumn{10}{|l|}{ Winters } \\
\hline Weekly fertigation & 97 & 92 & 4.72 & 4.57 & 25.2 & 2 & $43.5 \mathrm{~b}$ & 20.3 & $51.1 \mathrm{~b}$ \\
\hline No K control & 92 & 87 & 4.58 & 4.21 & 25.5 & 4 & $45.2 \mathrm{a}$ & 20.8 & $55.3 \mathrm{a}$ \\
\hline \multicolumn{10}{|l|}{ Contrast } \\
\hline Fertigation vs. no K control & NS & NS & NS & NS & NS & NS & * & NS & * \\
\hline Weekly fertigation & $127 \mathrm{a}$ & $120 \mathrm{a}$ & 4.50 & $5.42 \mathrm{a}$ & $26.5 \mathrm{~b}$ & 15 & $44.8 \mathrm{~b}$ & $20.2 \mathrm{~b}$ & $47.5 \mathrm{ab}$ \\
\hline No K control & $117 \mathrm{~b}$ & $109 \mathrm{~b}$ & 4.42 & $4.81 \mathrm{~b}$ & $27.7 \mathrm{a}$ & 11 & $45.9 \mathrm{a}$ & $21.7 \mathrm{a}$ & $49.8 \mathrm{a}$ \\
\hline \multicolumn{10}{|l|}{ Contrast } \\
\hline Fertigation vs. no K control & ** & $* *$ & NS & ** & ** & * & ** & ** & ** \\
\hline
\end{tabular}

${ }^{\mathrm{z}} \mathrm{A}$ seasonal total of $225 \mathrm{~kg} \cdot \mathrm{ha}^{-1} \mathrm{~K}$ was applied in both fertigation treatments.

yimensionless unit, lower value indicates more red.

${ }^{x}$ YS $=$ yellow shoulder.

wMeans within columns separated using Duncan's multiple range test, $p<0.05$.

NS, ${ }^{* * *}$ Nonsignificant or significant at $p<0.05$ or 0.01 , respectively.

beneficial, even in fields with high exchangeable soil K concentration. Not only was fruit color enhanced in all trials, but significant improvement in fruit yield and brix yield was also achieved at two sites as well. Fertigation appeared to be more effective than traditional Kapplication techniques (preplant or sidedress soil application, or injection into furrow irrigation water). In a series of earlier California field trials evaluating such $\mathrm{K}$ fertilization techniques (Hartz et al., 2001), no yield response was observed in any of 8 fields with exchangeable soil $\mathrm{K}>0.35 \mathrm{cmol} \cdot \mathrm{kg}^{-1}$; given this consistent lack of effect, no preplant or sidedress K treatments was included in the present study.

While fertigation may be a more effective means of $\mathrm{K}$ application, the changes in root distribution inherent in the use of a subsurface drip irrigation system may also restrict soil $\mathrm{K}$ availability, thereby increasing the potential for crop response to $\mathrm{K}$ fertigation. With subsurface drip irrigation, the active root zone is confined to the region wetted by the drip tape, which limits the soil volume effectively colonized by roots. Also, under California conditions, the surface soil usually remains dry throughout most of the season, restricting $\mathrm{K}$ uptake from the soil zone which typically has the highest exchangeable $\mathrm{K}$. The low late-season leaf $\mathrm{K}$ in the control treatment in the 2002 UCD and 2004 Woodland trials, despite relatively high exchangeable soil $\mathrm{K}$, lends credence to this theory.

The lack of a significant fruit yield response with $\mathrm{K}$ fertigation in the 2004 Winters trial (the field with the lowest exchangeable soil K) may have been attributable to a yield-limiting P deficiency. The lack of a yield response in the 2003 UCD trial was less easily explained; soil characteristics and all management practices were very similar to the 2002 trial, in which a large yield increase was observed. Leafanalysis in 2003 showed leaf $\mathrm{K}$ to be well within the established tissue sufficiency range; at both yield-responsive sites (2002 UCD and 2004 Woodland) late-season leafK was substantially lower, and at Woodland even midseason leaf $\mathrm{K}$ was marginal. The responsive sites also had the highest yield, and therefore the highest $\mathrm{K}$ demand, since fruit constitutes the primary $\mathrm{K}$ sink for the tomato crop (Adams, 1986).
Fruit yield increase was attributable primarily to increased fruit set; all fertigation treatments in which yield increase was observed were initiated during early fruit set. These results suggested that $\mathrm{K}$ fertigation should be concentrated before the end of fruit set to improve yield. With current determinate processing cultivars, effective fruit set terminates approximately 6 weeks before harvest in California conditions; $\mathrm{K}$ fertigation after that time may improve fruit quality, but would be unlikely to affect yield.

Foliar K application, although a common industry practice, was ineffective both in the present study, and the previous report of Hartz et al. (2001). Combining these studies, in a total of 5 field trials no yield or fruit quality benefit was found. Since commercial growers seldom utilize more than three foliar applications per crop, and five to six applications per crop were made in these trials, foliar $\mathrm{K}$ fertilization as currently practiced appears unlikely to be beneficial.

While fruit quality benefits of $\mathrm{K}$ fertigation were documented in all trials, there were no consistent differences between fertigation methods (continuous and weekly), rates, or timing. The reduced YS incidence in K fertigation treatments observed in both UCD trials was consistent with prior reports linking this disorder to $\mathrm{K}$ status (Hartz et al., 1999, 2001; Picha, 1987; Picha and Hall, 1981). The large cultivar effect on YS observed in the UCD trials confirmed the importance of cultivar selection in minimizing this disorder. The positive effects of K fertigation on the other fruit color parameters measured (blended color, $\mathrm{L}^{*}$ and hue) reinforced the significance of crop K status on tomato color. The lack of a K fertigation effect on SSC disagreed with the observation of Lachover (1972) that K fertilization increased SSC, but was consistent with the results of Hartz et al. (1999, 2001), who found no relationship between fruit SSC and soil K status or K fertilization. Lachover's study was conducted in pots, and in that limited soil volume rooting density and $\mathrm{K}$ dynamics may have differed substantially from a typical field setting.

Although fruit yield and/or quality improvements were observed in all field trials,
K fertigation would not necessarily have been economically justifiable; a grower would need to recover the cost of the fertilizer in higher yield and/or in a price premium for improved fruit quality. At current fertilizer prices, 225 to $250 \mathrm{~kg} \cdot \mathrm{ha}^{-1} \mathrm{~K}$ (the rate at which yield response was obtained in the 2004 and 2002 trials, respectively) in $\mathrm{KCl}$ form would cost about $\$ 140$ to $\$ 160 \mathrm{US}$, with $\mathrm{K}_{2} \mathrm{SO}_{4}$ significantly more expensive. With the contract price of processing tomatoes in California averaging about $\$ 100 / \mathrm{Mg}$, an average yield increase of at least $1.4 \mathrm{Mg} \cdot \mathrm{ha}^{-1}$ would be required to justify fertilization on yield improvement alone. Predicting which fields would be most likely to show yield increase or economically significant color improvement with $\mathrm{K}$ fertigation remains problematic. As a general rule, any of the following conditions may be indicators of potentially yield- or color quality-limiting conditions: low soil exchangeable $\mathrm{K}$ (on an absolute basis, or as a percent of base exchange), a restricted root zone due to limited capillary water movement from the drip tape, a field history of fruit color problems, or low leaf $\mathrm{K}$ concentration during fruit set.

\section{Literature Cited}

Adams, P. 1986. Mineral nutrition. p. 281-334. In: J.G. Atherton and J. Rudich (eds.). The tomato crop. Chapman and Hall, New York.

Cassman, K.G., D.C. Bryant, and B.A. Roberts. 1990. Comparison of soil test methods for predicting cotton response to soil and fertilizer potassium on potassium-fixing soils. Commun. Soil Sci. Plant Anal. 21:1727-1743.

Dumas, J.B. 1981. Sur les procédés de l'analyse organique. Annal. De Chimie., XLVII. p. 195-213.

Dumas, Y., C. Leoni, C.A.M Portas, and B. Bieche. 1994. Influence of water and nitrogen availability on yield and quality of processing tomato in the European Union countries. Acta Hort. 376:185-192.

Francis, D.M., S.A. Barringer, and R.E. Whitmoyer. 2000. Ultrastructural characterization of yellow shoulder disorder in a uniform ripening tomato genotype. HortScience 35:1114-1117.

Hartz, T.K., E.M. Miyao, and J.G. Valencia. 1998. DRIS evaluation of the nutritional status of processing tomato. HortScience 33:830-832. 
Hartz, T.K., E.M. Miyao, R.J. Mullen, M.D. Cahn, J.G. Valencia, and K.L. Brittan. 1999. Potassium requirements formaximum yield and fruit quality of processing tomato. J. Amer. Soc. Hort. Sci. 124:199-204.

Hartz, T.K., E.M. Miyao, R.J. Mullen, and M.D. Cahn. 2001. Potassium fertilization effects on processing tomato yield and quality. Acta Hort. 542:127-133.

Hartz, T.K., C. Giannini, R.O. Miller, and E.M. Miyao. 2002. Estimating soil potassium availability for processing tomato production. Commun. Soil Sci. Plant Anal. 33:1389-1400.

Johnson, C.M. and A. Ulrich. 1959. Analytical methods for use in plant analysis. Univ. Calif. Agr. Expt. Sta. Bul. 766:26-78.

Lachover, D. 1972. The effect of potassium on a 'roma'variety of processing tomato, with special reference to potassium uptake, yield and quality. Qual. Plant. Mater. Veg. XXI 3:165-177.

McGuire, R.G. 1992. Reporting of objective color measurements. HortScience 27:1254-1255.

Picha, D.H. 1987. Physiological factors associated with yellow shoulder expression in tomato fruit. J. Amer. Soc. Hort. Sci. 112:798-801.

Picha, D.H. and C.B. Hall. 1981. Influences of potassium, cultivar and season on tomato graywall and blotchy ripening. J. Amer. Soc. Hort. Sci
106:704-708.

Sah, R.N. and R.O. Miller. 1992. Spontaneous reaction for acid dissolution of biological tissues in closed vessels. Anal. Chem. 64:230-233.

Thomas, G.W. 1982. Exchangeable cations. p 159-165. In: A.L. Page et al. (ed.). Methods of soil analysis: Part 2. Chemical and microbiological properties. ASA Monogr. 9

Trudel, M.J. and J.L. Ozbun. 1971. Influence of potassium on carotenoid content of tomato fruit. J. Amer. Soc. Hort. Sci. 96:763-765.

Widders, I.E. and O.A. Lorenz. 1979. Tomato root development as related to potassium nutrition. J. Amer. Soc. Hort. Sci. 104:216-220. 